mer's test, and Fehling and Barreswil's solutions, and other modifications of the copper test, may be drawn*:-

1. That if the urine contain chloride of ammonium (even in very small quantity), urate of ammonia, or other ammoniacal salts, the suboxide of copper would not be precipitated if only a small quantity of sugar were present.

2. That unless there be a considerable quantity of one of the above salts present (in which case the blue colour will remain), the mixture will change to a brownish hue upon boiling, but no opalescence or precipitate of suboxide of copper will occur. When only a moderate amount of sugar is present, I have been unable to obtain a precipitate, under these circumstances, by the addition of potash to the solution, and prolonged boiling. By observation 8, it appears that a specimen of urine exhibiting this reaction may contain a large quantity of sugar, as ascertained by the yeast test.

3. That in many cases in which the precipitation of the suboxide is prevented by the presence of ammoniacal salts, the addition of potash to the solution, and subsequent boiling, will cause the production of a precipitate with the evolution of ammoniacal fumes. Hence care should always be taken that there is a considerable excess of free alkali present.

4. When only small quantities of sugar are present in the urine, and the precipitate of suboxide of copper is not decided, the fermentation test should be resorted to.

Upon treating different specimens of diabetic urine with Trommer's test, or its modifications, it has often been noticed that in one case the precipitate is produced as soon as the mixture reaches the boiling point, or even before; while in other instances it is necessary to keep it in active ebullition for some minutes, before any precipitate is produced. This circumstance receives explanation from the facts above detailed with reference to the presence of ammoniacal salts; and other anomalous results, which must have occurred to many in the habit of employing this test, become explained.

As a test for diabetic or grape-sugar, if proper precautions be observed, much greater dependance can be placed on this test than by simply boiling with liquor potassæ. Specimens of urine in which sugar is suspected to be present, and no decided precipitate of suboxide (which must be carefully distinguished from phosphate + ) occurs, should be carefully fermented with yeast before any conclusion is arrived at.

On Testing for Sugar when only Traces arc Present. In fluids which are suspected to contain only mere traces of sugar, it is necessury to separate some of the other constituents before applying the test. The plan recommended by M. Leconte is the following:-Excess of acetate of lead is added, and the precipitate separated by filtration. The solution is concentrated by evaporation, treated with ammonia, and again filtered. The copper test is then applied. The objections to applying the reduction test to solutions containing ammonia has been already discussed. It is better to employ carbonate of potash, or soda, instead of ammonia. The excess of lead salt may also be removed from the filtered solution by passing sulphuretted hydrogen. The precipitate of sulphuret of lead is removed by filtration, and the liquid, after evaporation to a small bulk, may be tested.

Another plan recommended by M. Leconte is to treat the urine with acetic acid, and evaporate it to about the fifth of its bulk; it is then treated with alcohol, and after filtration from the salts, etc., the alcoholic solution is evaporated and tested. This plan is free from the objection that ammonia may cause the destruction of the sugar where only traces are present.

The Yeast Test. This is one of the most satisfactory tests for the presence of sugar, and if employed with proper care can hardly fail in its results. Two test tubes, of the same form, and of equal size, are to be taken. One is nearly filled with water, and into the other a corresponding quantity of the urine is to be poured. An equal amount of yeast is now to be added to the liquids in the tubes, and after pouring in just sufficient fluid to fill the tubes, the thumb is to be carefully placed over the opening, and the tube inverted in a small cup of mercury. The best plan, however, is to cut out a little india.rubber pad, slightly larger than the upper extremity of the tube. When the tubes have been filled up to the brim with a little water, the pad is allowed to float on the surface; next a little cup or

- Professor Brücke has recently drawn attention to the action of ammonis in preventing the precipitation of the suboxide of copper, and other points connected with this subject. Probably he had not seen the results just given, which were obtained in 1852. (Mled.-Chir. Review, Jan. 1853, vol. xi,
p. 113.) solubility in ammonia. beaker is inverted, and carefully placed over the end of the tube. The india-rubber being pressed against the open end, the fluid is prevented from escaping. The whole may be inverted, and a little mercury having been poured into the beaker, the india-rubber may be removed, with forceps, without any escape of the fluid. The tubes may be supported in position by a wire stand. Both tubes are then to be exposed, for a few hours, to a temperature of from $80^{\circ}$ to $90^{\circ}$, and the comparative size of the bubble of gas in the upper part of each may then be noted. If an appreciable quantity of sugar be present, the bubble of gas in the tube containing the urine will be many times larger than that in the tube which contains the yeast and water. In the latter tube the bubble of gas merely arises from the small quantities of air previously mixed with the yeast, becoming disengaged, and floating to the surface. Fermentation, when carefully performed, is positive evidence of the presence of sugar, although it does not indicate the kind of sugar present.

Maumene"s Test. A little woollen rag, as merino, is cut into strips, and soaked for four or five minutes in a solution of perchloride of tin (one part of the perchloride to two parts of water). The slips are then dried over the water bath. A drop of the urine suspected to contain sugar is allowed to fall on a small slip of the prepared merino, which is then dried, and exposed to the dull red heat of a spirit lamp. If a trace of sugar be present, a black spot is produced.

Bismuth Test. Bottger has lately proposed a new test for sugar. This consists in adding first of all potash, then a small quantity of subnitrate of bismuth; lastly, the mixture is boiled. If sugar is present, the oxide is reduced to metallic bismuth, which is precipitated in the form of a black powder. It has been asserted that sulphuret of bismuth is formed, but this seems not to be the case. Brücke shows that this test is more delicate than Trommer's (or the modification of it by Fehling); and he finds that the black precipitate is produced to some extent in specimens of healthy urine. The bismuth test may be also applied thus. A solution of carbonate of soda ( crystallised carbonate 1 part, water 3 parts) is prepared, and a certain quantity added to an equal amount of the urine. A little basic nitrate of bismuth is then added, and the mixture heated to the boiling point. If sugar be present, a black precipitate is produced.

[To be continued.]

\section{bitional Communications.}

\section{A CASE OF SUDDEN DEATH, WITHOUT ANY ADEQUATE POST MORTEM APPEARANCES.}

By J. Zachariah Laurence, F.R.C.S., M.B.Lond., Surgeon to the South London Ophthalmic Hospital, and the Hospital for Paralysis.

[ Communicated to the Harveian Socicty, May Srd, 1860.]

ON the 24th of April, the housemaid in a medical man's family was sent out to fetch the beer, when, within ten minutes afterwards, she was found by the cook lying apparently dead in the scullery. I saw her myself in about another quarter of an hour. There was no action of the heart, no breathing, no sensibility; the body was still warm; the face livid and swollen. The only symptoms observed during life were that, for about a week before her death, she suffered from earache, appeared unusually indolent and stupid, and at times complained of faintness.

Post Morteir Examination, about twenty-two hours after death. The body was large, finely developed, very fat. Cadaveric rigidity was strongly marked in the limbs, and the firmly clenched lower jaw. The left ventricle of the heart was filled with dark fluid blood, and was firmly contracted; the right was flaccid and collapsed, containing but a very small clot. The muscular substance was apparently normal. The heart was altogether small in proportion to the body. The lungs were slightly congested. Between the two openings of the stomach was an oblong patch of fine punctiform florid injection in the lines of the rugæ. It contained an undigested meal of some stewed steak, of which the patient had partaken shortly before death. No poison of any kind was found in a careful analysis instituted by Mr. Rodgers, at the request of the jury. The intestines were not opened; their peritoneal coat was covered with congested veins, but in no very marked degree. The 
liver was somewhat congested. The spleen was soft and congested. The kidneys were very much congested. The uterus, ovaries, and Fallopian tubes, were much congested. (She was menstruating at the time of her death.) The superficial vein of the brain, as those of the medulla oblongata, were turgid with dark blood. There was a slight amount of serum beneath the arachnoid (which was somewhat opaque here and there), especially in the basilar fossa. The brain-substance itself was perfectly normal. The larynx, trachea, and œosophagus were healthy, and contained no traces of any food in them.

REMARKS. This case is interesting in medical jurisprudence, in two points of view : first, for the unusual precision with which the entire suddenness of the death could be fixed; second, for the total absence of any post mortem appearances usually found in cases of sudden death (such as heart-disease, bursting of aneurisms, etc.). In the Annales d'Hygiène Publique for 1838 will be found a paper by Devergie entitled "De la Mort Subite", in which, with other causes of sudden death, he signalises that by syncope, which he had found to occur three times in forty cases. Dr. Tourdes reported to the scientitic congress meeting held at Strasbourg in 1842, that he had met with this cause of sudden death once in twenty-six cases. It appears to be identical with the "idiopathic asphyxia" of Chevalier. Devergie, in the paper above referred to, gives the following as the post mortem appearances he found in his three cases of death from syncope. " 1 . The absence of all congestion of the organs. 2. The normal state of all the organs. 3. The existence of about an equal quantity of blood in the right and left cavities of the heart, regard being had to their respective sizes. 4. Perhaps the coagulation of the blood in the fibrinous state." As another cause of sudden death Devergie further indicates that arising from cerebral conges tion, which may, he says, be limited to the meninges. In illustration of this, he relates the case of a man aged 63 , who, having only complained of a slight headache in the morning, took a good breakfast, after which he slept for two bours, and, on awaking, suddenly died. No congestion of the brainsubstance was found; there was an old apoplectic clot in the left corpus striatum; there was great congestion of the meninges of the brain and spinal cord.

Directing attention now to the condition of the stomach in our case, we may naturally ask ourselves whether the undigested meal, or the patch of injection on its coats, may have had anything to do with the cause of death? As regards the latter, no one would, I think, after the researches of Dr. Yellowly and others, attach any great independent value to mere vascularity of the gastric mucous membrane. The undigested meal may perhaps possess rather more significance. The deceased seems to have been in the habit of "bolting" her meals very rapidly. The stewed steak found in her stomach, I am informed, she partook of stealthily - and therefore probably hastily-before her proper dinner, shortly before death.

Dr. Taylor, at p. 152 of the second edition of his work on Poisons, has cited several instances of persons dying quite suddenly, with nothing to account for death but a distension of the stomach ; but I noticed no such distension in the present case. We may not unreasonably connect these conditions of the stomach with the syncopal affection. Professor Weber has shown that irritation of the pneumogastrics will stop the heart's action; and my distinguished friend and colleague, Dr. Brown-Séquard, has further shown that crushing the semilunar ganglia has a similar effect on the heart, provided the great splanchnic and pneumogastric nerves remain intact.

I should feel disposed to regard the present case as one of a mixed nature, presenting some of the signs of syncopal asphyxia, others of apoplexy. Explain it, however, as we may, it still remains one of considerable interest, proving as it does that, under certain (as yet very imperfectly understood) circumstances, death may occur suddenly, without leaving any traces at all adequate (in our present state of knowledge) to account for the fatal result.

\section{CONNECTION BETWEEN CANCER AND FRAGILITAS OSSIUM.}

By W. McCheane, Esq., Liverpool.

THE following case may perhaps be worth recording, as illustrative of the connection between cancer and fragilitas ossium.

R. W., a female, aged 68 , had been under my care for some time for a scirrhous tumour in the right breast, from which situation a similar tumour was formerly removed. Of late, she had been altogether confined to bed with guawing pains in the limbs, but more especially in the lower extremities, and in a state of general cachexia.

On the 1st instant, during a fit of coughing, she felt a something give way in the region of the right hip, which sensation was immediately followed by severe pain in the part and down the whole limb. On examination, I found a fracture of the neck of the thigh-bone, which injury she survived only a week.

Some months ago she fractured two ribs with lifting a pan off the fire; the bones, however, readily united.

\section{Cramsactions of 通rand hes.}

\section{BIRMINGHAM AND MIDLAND COUNTIES B RANCH.}

ON THE PATHOLOGY OF LEAD-COLIC.

By Willoughby F. WADE, M.B., Physician to the Queen's Hosnital and to the General Dispensary; Professor of the Practice of Physic in the Queen's College, Birmingham.

[Read April 19th, 1860.]

THE received opinion that this painful disorder depends upon some perverted action of the colon, as its name implies, has already had its antagonists. When we come to inquire a little more closely what this perverted action is, we find that no satisfactory answer can be given. Some contend for an empty and contracted condition of the gut, others for a distension by gas or fæces.

Dr. Copland says that in his cases distension was as frequent as retraction, owing evidently to inflation and fæcal engorgement of the colon, the cour'se of which could be distinctly traced under the abdominal parietes. De Haen and Merat found contraction of the colon and cæcum in all the cases they examined. But, as Dr. Watson judiciously remarks, "with regard to the contraction of the large intestine in these cases, we must not be too ready to attribute it to spasm, for the bowel, when empty, is apt to be contracted." Andral details six cases in which no such contractions were found. Indeed Andral, Louis, and Sir George Baker, concur in describing the intestines as being normal throughout their whole extent. I doubt very much whether an unopposed contraction of a hollow muscular canal can be attended with pain. It is the vain endeavour to shorten the muscular tissue, and the resistance offered by an incompressible material, that causes the pain in biliary calculus and ordinary crapulous or flatulent colic. An empty intestine might, I think, go on contracting till its calibre was obliterated before it produced pain. 'The after-pains of labour do not offer any necessary objection to this view; for the contraction of one layer of fibres can be well resisted by the large mass of inactive ones. Besides, they often depend on the presence of clots. On the other hand, did the pain of lead-colic depend upon the presence of flatus, I cannot conceive how it is that this should not, in such cases, be readily removed, for a time at least, by opiates and carminatives, as happens in ordinary flatulence. If, again, it depended upon retained fæces, the removal of them should remove the pain. But the operation of the bowels is by no means necessarily followed by this relief. It is, indeed, true that the two often coincide, but this is quite as easily explicable in another way, as we shall see directly. The retraction of the abdominal parietes, so constantly noticed in this form of the complaint, is by no means so constantly observed in other varieties of colic.

The pathology of lead-colic is then, I submit, unsatisfactory and vague as at present taught.

Various pathologists of distinction have been disposed to refer the symptoms to cramp of the external abdominal muscles, instead of to the intestines at all. Giacomini first broached this notion, and M. Briquet of La Charite has more lately revived this view, which he supports with skill and vigour. The existence of cramp in these muscles has been recognised by those who are entirely committed to the generally accepted pathology. Thus Dr. Copland says, " the voluntary muscles often become so sore that they cannot bear the slightest pressure; and the pain frequently alternates between the stomach and bowels and the external muscles." Besides 